\title{
Consistency of expert-based preference matrices
}

\author{
Martin Gavalec ${ }^{\mathrm{a}}$, Karel Mls ${ }^{\mathrm{a}}$ \\ ${ }^{a}$ University of Hradec Králové, Faculty of Informatics and Management, Rokitanského \\ 62, 50003 Hradec Králové, Czech Republic
}

Keywords: preference matrix, AHP decision making, cyclic consistency, consistent matrix

2000 MSC: 90C15

\section{Cyclic inconsistency of a preference matrix}

In AHP approach to multi-criteria decision problem, the relative importance of alternatives is computed from preference matrices, which come from experience and can possibly be inconsistent.

An algorithm for computing a consistent approximation of a given preference matrix by digraph method is described in this paper. We start with an analysis of the inconsistency of a given preference matrix. The first type of inconsistency is caused by so-called inconsistency cycles. The inconsistency of this type is removed by computing the strongly connected components in the associated digraph and a small modification. If the modified matrix is cyclic consistent, i.e. it contains no inconsistent cycles, or if some of the entries of the matrix are missing, then a consistent approximation is computed. The computational complexity of the algorithm is $O\left(n^{2}\right)$.

Preference matrix $A$ is called cyclic inconsistent, if there is a cycle

$$
i_{1}, i_{2}, \ldots, i_{r}, i_{r+1}=i_{1}
$$

of length $r \geq 2$ of indices in $N$ (called: inconsistent cycle in $A$ ) such that the inequalities

$$
a\left(i_{k} i_{k+1}\right) \geq 1 \quad \text { for every } \quad k=1,2, \ldots, r
$$

Email addresses: martin.gavalec@uhk.cz (Martin Gavalec), karel.mls@uhk.cz (Karel Mls) 
hold, and at least one of the inequalities is strict. Matrix $A$ is cyclic consistent, if every cycle in $A$ is consistent, i.e. $A$ contains no inconsistent cycles. Examples of inconsistent cycles of length $r=3$ are shown in Figure 1.
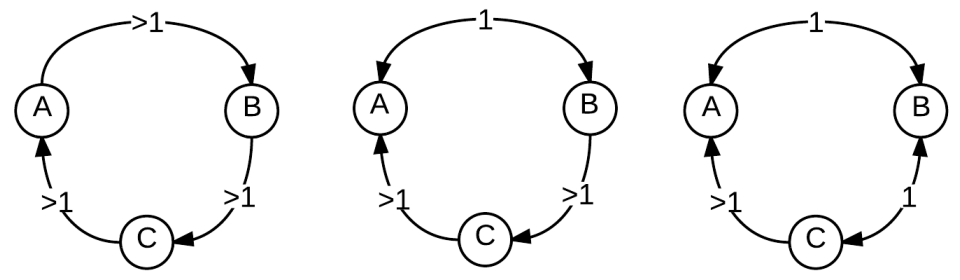

Figure 1: Possible types of cyclic inconsistency with $r=3$

Theorem 1.1. If a complete preference matrix A contains an inconsistent cycle of length $r>3$, then A also contains an inconsistent cycle of length 3.

Corollary 1.2. The cyclic consistency of a complete preference matrix can be recognized in time $O\left(n^{3}\right)$, by verifying all index cycles of length 3 for the inconsistency.

Theorem 1.1 is illustrated by Figure 2 below.
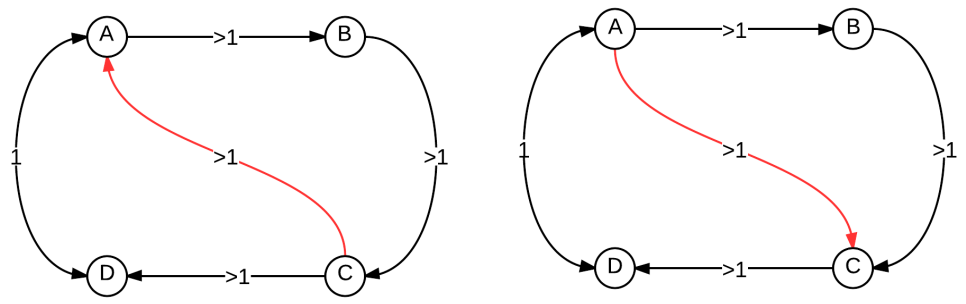

Figure 2: Cyclic inconsistency with $r=4$

The preference digraph $\mathcal{D}=(V(A), E(A))$ of a given preference matrix $A$ is defined as follows

$$
\begin{aligned}
& V(\mathcal{D})=\{1,2, \ldots, n\} \\
& E(\mathcal{D})=\left\{(i, j) ; a_{i j} \geq 1\right\} \\
& E^{+}(\mathcal{D})=\left\{(i, j) ; a_{i j}>1\right\}
\end{aligned}
$$

The edges in $E^{+}(\mathcal{D})$ are called strong preference edges. 
Theorem 1.3. Preference matrix $A$ is cyclic consistent if and only if every cycle $C$ in $\mathcal{D}(A)$ contains no strong preference edges, i.e. $C \cap E^{+}(\mathcal{D})=\emptyset$.

Theorem 1.4. Preference matrix $A$ is cyclic consistent if and only if every strongly connected component $\mathcal{K}$ in $\mathcal{D}(A)$ contains no strong preference edges, i.e. $(\mathcal{K} \times \mathcal{K}) \cap E^{+}(\mathcal{D})=\emptyset$.

A possible treating method (3-cycle method, for short) is based on Theorem 1.1.

\section{3-cycle method}

1 find all inconsistent cycles of length 3 in $A$

2 change all preferences in the cycles to 1

3 repeat 1 - 2 until there is no inconsistent cycle of length 3 in $A$

The repetition of steps 1 and 2 in the 3-cycle method is necessary, because new inconsistent cycles can be created by treating the cyclic inconsistency by cycles of length 3 (see Figure 3).
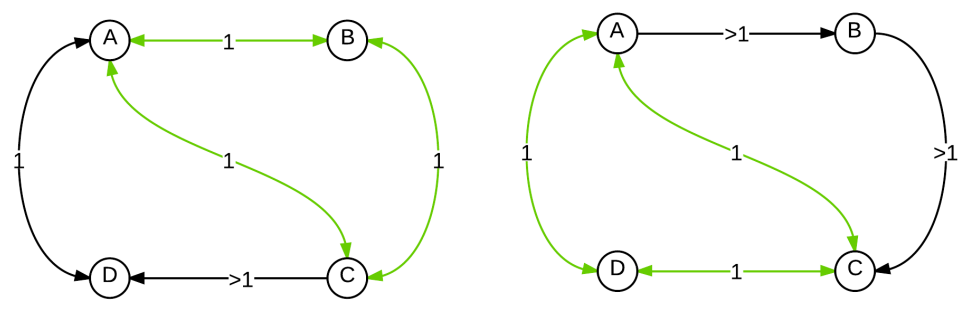

Figure 3: Creating new inconsistent cycles of lenth $r=3$

The above disadvantage is not present at another method for treating the inconsistency (SCC method, for short) which works with strongly connected components in the preference digraph $\mathcal{D}(A)$. The method is based on Theorem 1.4.

\section{SCC method}

1 find all strongly connected components in digraf $\mathcal{D}(A)$

2 change all preferences within the strongly connected components to 1 
Theorem 1.5. If matrix $A^{\prime}$ is created from preference matrix $A$ by $S C C$ method, i.e.

$a_{i j}^{\prime}=\left\{\begin{array}{ll}1 & \text { for } i, j \in \mathcal{K} \text { in every strongly connected component } \mathcal{K} \text { of } \mathcal{D}(A) \\ a_{i j} & \text { otherwise }\end{array}\right.$, then $A^{\prime}$ is reciprocal and cyclic consistent.

The cyclic consistent matrix $A^{\prime}$ computed by the SCC algorithm is called the cyclic consistent approximation of $A$.

\section{Computing consistent preferences}

Preference order $\mathcal{P}(A)$ induced by $A$ is defined as follows: if inequalities $a\left(i_{k} i_{k+1}\right) \geq 1$ with $k=1,2, \ldots, r-1$ hold for some sequence $i=$ $i_{1}, i_{2}, \ldots, i_{r}=j$, then $(i, j) \in \mathcal{P}(A)$.

Theorem 2.1. If a preference matrix $A$ is cyclic consistent, then $\mathcal{P}(A)$ is a uniquely determined linear order of alternatives (up to permutations of equivalent preferences).

Theorem 2.2. If $A$ is a preference matrix and $A^{\prime}$ is its cyclic consistent approximation, then the linear order $\mathcal{P}\left(A^{\prime}\right)$ of alternatives is equal to the order of strictly connected components in preference digraph $\mathcal{D}(A)$.

\section{Algorithm ConsistApprox}

1 Input: preference matrix $A$

2 compute preference digraph $\mathcal{D}(A)$

3 compute cyclic consistent matrix $A^{\prime}$ by SCC method

4 compute linear order $\mathcal{P}\left(A^{\prime}\right)$ induced by the order of strongly connected components in digraph $\mathcal{D}(A)$

5 for any component $\mathcal{K}$ and its successor $\mathcal{L}$ substitute values $a^{\prime}{ }_{i j}$ with $i \in \mathcal{K}, j \in \mathcal{L}$ by their common geometric mean $\tilde{a}_{i j}$

6 extend the 'overdiagonal block' values in matrix $\tilde{A}$ using the reciprocity and consistency condition

7 Output: reciprocal and consistent approximation matrix $\tilde{A}$

Theorem 2.3. Algorithm ConsistApprox works correctly and for every $n \times n$ preference matrix $A$ the algorithm computes a consistent aproximation $\tilde{A}$ in $O\left(n^{2}\right)$ time. 
Remark 2.4. Algorithm ConsistApprox can easily be modified also for the case of missing values in the input preference matrix $A$. In the modification, the missing input values create no edges in the preference digraph $\mathcal{D}(A)$.

Theorem 2.5. If $A$ is a preference matrix with missing values and if the order of strongly connected components in the preference digraph $\mathcal{D}(A)$ is linear, then algorithm ConsistApprox with input matrix A works correctly, and the algorithm computes a consistent aproximation $\tilde{A}$ in $O\left(n^{2}\right)$ time.

\section{Acknowledgement}

The support of the Czech Science Foundation project \# 14-02424S is gratefully acknowledged. 